\title{
Tratamiento regenerativo con células madre mesenquimales provenientes de la gelatina de Wharton de cordón umbilical en la úlcera crónica por dermolipectomía
}

\author{
Regenerative treatment with umbilical cord mesenchymal stem cells from Wharton's jelly \\ in chronic ulcer caused by dermolipectomy
}

\author{
César M. Mejía-Barradas, Jaime E. Cázares-Montañez², Ángel Guerra-Márquez³, Víctor G. Hernández- \\ Chávez ${ }^{4}$, Julio R. Cáceres-Cortés ${ }^{1}$ y Gisela Gutiérrez-Iglesias ${ }^{1 *}$ \\ ${ }^{1}$ Sección de Estudios de Posgrado e Investigación, Escuela Superior de Medicina, Instituto Politécnico Nacional; ${ }^{2 H o s p i t a l ~ R e g i o n a l ~ T l a l n e p a n t l a, ~}$ \\ Instituto de Seguridad Social del Estado de México y Municipios; ${ }^{3}$ Banco Central de Sangre, Centro Médico Nacional La Raza, Instituto Mexicano \\ del Seguro Social; ${ }^{4}$ Escuela Nacional de Ciencias Biológicas, Instituto Politécnico Nacional, Unidad Profesional Lázaro Cárdenas. Ciudad de México, \\ México
}

\begin{abstract}
Resumen
Introducción: Los procedimientos para retirar adiposidades y piel, como la dermolipectomía, pueden desarrollar heridas difíciles de sanar mediante tratamientos convencionales. Se ha señalado que es posible utilizar las células madre mesenquimales en el tratamiento regenerativo en heridas, en virtud de su multipotencialidad, baja inmunogenicidad, capacidad moduladora de inflamación y procesos modeladores de tejidos. Caso clínico: Paciente con dehiscencia en úlcera crónica secundaria a dermolipectomía, sometida a tratamiento cutáneo con células madre mesenquimales. Se indujo formación de cicatriz y neovascularización, así como la disminución de leucocitos infiltrados y citocinas proinflamatorias. Se propone a las células mesenquimales como una alternativa interesante para el tratamiento de lesiones postoperatorias.
\end{abstract}

PALABRAS CLAVE: Úlcera crónica. Dermolipectomía. Células madre mesenquimales. Medicina regenerativa. Cicatrización. Inflamación.

\begin{abstract}
Background: Procedures to remove adiposities and skin, such as dermolipectomy, can develop wounds that are difficult to heal by conventional therapies. Mesenchymal stem cells are indicated as potential candidates for regenerative therapy in wounds, due to their multipotentiality, low immunogenicity, modulating capacity of inflammation and tissue modeling processes. Case report: Patient with dehiscent chronic ulcer secondary to dermolipectomy, who received cutaneous treatment with mesenchymal stem cells. The therapy induced scar formation and neovascularization, as well as the decrease of infiltrated leukocytes and proinflammatory cytokines. Mesenchymal cells are proposed as an interesting alternative for the treatment of postoperative lesions.
\end{abstract}

KEY WORDS: Chronic ulcer. Dermolipectomy. Mesenchymal stem cells. Regenerative medicine. Scarring. Inflammation.

\author{
Correspondencia: \\ *Gisela Gutiérrez-Iglesias \\ Plan de San Luis y Díaz Mirón, s/n \\ Col. Casco de Santo Tomás, Del. Miguel Hidalgo \\ C.P. 11340 , Ciudad de México, México \\ E-mail: iglesiasgg@yahoo.com
}

Fecha de recepción: 07-06-2018

Fecha de aceptación: 24-08-2018

DOI: $10.24875 / C I R U .18000515$
Cir Cir. 2019;87(S1):8-16

Contents available at PubMed www.cirugiaycirujanos.com 


\section{Introducción}

La dermolipectomía es un procedimiento quirúrgico con fines funcionales y estéticos que consiste en retirar excesos de piel y tejido adiposo en brazos y abdomen ${ }^{1,2}$. Este procedimiento posee una tasa de complicaciones generales de $45.8 \%$, entre las que se encuentran los seromas (23.6\%), infecciones (13.9\%), hemorragias $(11.1 \%)$, hematomas $(6.9 \%)$, necrosis de la piel $(6.9 \%)$ y necrosis umbilical $(4.2 \%)$; de éstas, un total de $11.1 \%$ requiere reoperación ${ }^{3}$. Este procedimiento estético, como cualquier práctica quirúrgica, se acompaña de lesiones persistentes o recurrentes debido al desarrollo de dehiscencias que interfieren con la cicatrización en $7.3 \%$ de los pacientes, lo que ocasiona úlceras crónicas de difícil tratamiento 4 . Estas lesiones suelen complicarse con diferentes alteraciones metabólicas, como diabetes, obesidad, hipertensión y desnutrición, entre otros ${ }^{5-7}$.

El tratamiento convencional de este tipo de lesiones consiste por lo general en la aplicación de parches hidrocoloides, fármacos, operaciones o injertos de piel autólogos; sin embargo, éstos no son del todo efectivos y pueden presentar efectos adversos y secundarios $^{8-13}$. Como alternativa se plantea el uso de células madre mesenquimales (CMM) como tratamiento celular para regenerar y cicatrizar órganos y tejidos ${ }^{14}$, ya que tienen la capacidad de autorrenovarse y diferenciarse en osteoblastos, condrocitos, adipocitos, células musculares y neurocitos ${ }^{15,16}$. Hasta ahora, el estudio de las CMM se centra en las provenientes de médula ósea como norma de referencia, aunque en fecha reciente se han propuesto novedosas fuentes que no implican métodos invasivos para su obtención con potenciales fines terapéuticos.

Una fuente accesible de CMM es la gelatina de Wharton (CMMWG) de cordón umbilical, que representa un tejido rico en células madre con alta capacidad de autorrenovación y diferenciación múltiple, y dado que se desecha después del parto su uso no representa restricciones éticas ${ }^{17,18}$. Por otra parte, las CMMGW tienen baja inmunogenicidad debido a que no activan la proliferación de células $T$, al no expresar el complejo principal de histocompatibilidad clase II (MHCII) ni moléculas coestimuladoras como CD40, CD80 y CD86, que son necesarias para la activación de las células $T^{19}$. Estudios clínicos demuestran que al administrar CMMGW la proliferación de linfocitos se suprime y además no activa al $\mathrm{MHC}^{20}$. Por esta razón, el rechazo después del trasplante del injerto celular es mínimo o nulo, incluso entre individuos no emparentados ${ }^{21}$. Además, las $\mathrm{CMM}$ presentan un efecto inmunomodulador en los linfocitos $T$ y $B$, células dendríticas y NK, ya que se puede inhibir la proliferación de las células inmunitarias y reducir la secreción de citocinas y la presencia de los subtipos de células infiltradas en tejido $20,22-25$, lo cual atenúa la inflamación y promueve la cicatrización de las lesiones.

Estas características y propiedades de las CMMGW alogénicas proporcionan una ventaja para la regeneración de tejidos y lograr así el cierre de heridas y evitar el rechazo. El presente trabajo tuvo como objetivo emplear el tratamiento de células madre mesenquimales como una alternativa para la resolución de úlceras persistentes en las que los procedimientos convencionales no han permitido la cicatrización. Las CMMGW de cordón umbilical, aisladas y trasplantadas en heridas de gran persistencia, evitarán el rechazo inmunológico y recobrarán la capacidad de recuperación e inmunorregulación, así como la remodelación del tejido dañado de úlceras secundarias a operaciones. Esto representa una novedosa alternativa terapéutica para el tratamiento de úlceras y lesiones de difícil atención.

\section{Materiales y métodos}

\section{Caso clínico}

Mujer de 41 años de edad con úlcera crónica abdominal transversal de tres meses derivada de dermolipectomía estética. La herida presentaba una extensión de $6 \times 7 \mathrm{~cm}$ y profundidad de $1 \mathrm{~cm}$ con hiperqueratosis, bordes engrosados, aperlados, pobre cicatrización espontánea, y datos de infección persistente: eritema, exudado purulento, dolor y dehiscencia. La lesión respondió de forma discreta a los tratamientos como yodopovidona, peróxido de hidrógeno o soluciones hiperoxidantes. Después del tratamiento con antibióticos orales se valoró a la paciente como elegible para recibir tratamiento celular. Una vez informada de riesgos y beneficios consintió mediante su firma para participar en el protocolo de investigación del uso de CMM como tratamiento regenerativo proporcionado por el personal científico de la Escuela Superior de Medicina del Instituto Politécnico Nacional en colaboración con el Instituto de Seguridad Social del Estado de México y Municipios (ISSEMYM), con la autorización del comité de ética e investigación con registro CIS Y CEIS/0118 P.I./15. 
Cirugía y Cirujanos. 2019;87(S1)

Tabla 1. Oligonucleótidos iniciadores para PCR en tiempo real

\begin{tabular}{llll}
\hline Gen & Referencia NCBI & Secuencia de oligonucleótidos & Reverse \\
\cline { 3 - 4 } & & Forward & actgtgaggaggggagattc \\
\hline GAPDH & NM_002046.3 & tcaacgaccacttgtcaag & caagacttgtggggctgag \\
CD105 & NM_000118.2 & tgccactggacacaggataa & aaccacgttgatatcttggtca \\
CD73 & NM_001204813.1 & cctctcaaaatggacgaggt & gccctcacacttgaccagtt \\
CD90 & NM_006288.3 & aggacgagggcacctacac & ttctctctaagaaacaccatcacct \\
HLA-DR & NM_019111.4 & tcttcatcatcaagggattgc & tccaccgtttccgtgtaat \\
CD34 & NM_001025109.1 & tggctattcctgatgaatcg & ggcattaaggtaggcatctga \\
CD45 & NM_002838.3 & ccattgacaaccaccctca &
\end{tabular}

\section{Obtención de células madre mesenquimales de gelatina de Wharton y su aplicación}

Las CMM se aislaron a partir de gelatina de Wharton de cordones umbilicales de madres saludables que llegaron a término de gestación a las 38 a 40 semanas; estos cordones se seleccionaron de forma previa y los donó el Banco de Cordón Umbilical del Hospital la Raza del IMSS (que cuenta con certificación de seguridad biológica). Para obtener los cultivos celulares, los cordones umbilicales se lavaron y procesaron en el Laboratorio de Medicina Regenerativa y Estudios en Cáncer de la Escuela Superior de Medicina del IPN, donde se aplicaron dos digestiones enzimáticas consecutivas: colagenasa tipo 2 (Sigma-Aldrich, EE.UU.) al $1 \%$ en PBS y tripsina (Gibco ${ }^{\circledR}$, EE.UU.) al $0.1 \%$, ambas con agitación constante a $37.5^{\circ} \mathrm{C}$ durante 60 y $30 \mathrm{~min}$, respectivamente. La mezcla digerida se cultivó en placas de $100 \mathrm{~mm}$ de diámetro en medio DMEM-F12 al 10\% de suero fetal bovino y $1 \mathrm{x}$ de Antibiotic/Antimicotic (Gibco ${ }^{\circledR}$, EE.UU.), a $37^{\circ} \mathrm{C}, 5 \%$ de $\mathrm{CO}_{2}$ y atmósfera saturada de humedad. Al alcanzar confluencia de 80 a $90 \%$, las células se desprendieron y resembraron sin utilizar más de un séptimo pase. Las CMM se caracterizaron y seleccionaron con base en los criterios de la International Society for Cellular Therapy ${ }^{26}$. Las CMM listas para la aplicación se suspendieron en solución salina isotónica estéril para administrarse de manera intradérmica en los bordes y el lecho de lesión a razón de $100000 \pm 10000$ células $/ \mathrm{cm}^{2}$ de herida ${ }^{27}$, previos lavado y desinfección de la zona de aplicación. La lesión se revisó y cubrió con apósitos estériles cada día. La aplicación celular se repitió a los siete días.

\section{AdHERENCIA Y MORFOLOGÍA SIMILAR A FIBROBLASTOS}

Se seleccionaron las células que presentaron en cultivo adherencia y morfología similar a fibroblastos con base en los criterios de la ISCT.

\section{DIFERENCIACIÓN OSTEOGÉNICA Y ADIPOGÉNICA}

Se mantuvieron placas de cultivo tomadas de diferentes pases durante 21 días con Stempro ${ }^{\circledR}$ Osteogenesis Differentiation Kit (Gibco ${ }^{\circledR}, \mathrm{EE} . U \mathrm{UU}$.). Con posterioridad, las células se fijaron con formol al $10 \%$, y se tiñeron con rojo de alizarina 10 min para identificar depósitos de calcio. De igual manera, otros cultivos celulares se mantuvieron con Stempro ${ }^{\circledR}$ Adipogenesis Differentiation Kit $\left(\mathrm{Gibco}^{\circledR}\right.$, EE.UU.) durante 21 días. A continuación, las células se fijaron con formol al $10 \%$ y se tiñeron con rojo oleoso en isopropanol al $60 \%$ para evidenciar vacuolas grasas.

\section{EXPRESIÓN GÉNICA DE ANTÍGENOS DE SUPERFICIE}

La expresión génica para los marcadores de superficie de las CMM se evaluó con RT-PCR cuantitativa en tiempo real. El RNA total se extrajo a partir de $1 \times 10^{6}$ células en cultivo y también de leucocitos de $5 \mathrm{ml}$ de sangre periférica, como control de expresión hematopoyética, mediante el protocolo de extracción TRIzo ${ }^{\circledR}$ reagent (Invitrogen, EE.UU.). EI DNAc para la PCR se sintetizó con el First Transcriptor Strand cDNA Synthesis Kit (Roche Applied Science, Alemania). El diseño específico de gen de los oligonucleótidos iniciadores o cebadores de la reacción (primers) se realizó a través de Universal ProbeLibrary System Assay Design de www.lifescience.roche.com (Tabla 1). Para la reacción de amplificación se utilizó LightCycler ${ }^{\circledR} 480$ 
SYBER Green I Master kit (Roche, Alemania) en un LightCycler 1.5 Thermoclycler (Roche, Alemania). Se utilizó el método de $\Delta \Delta$ Ct para el análisis de la expresión relativa con el gen normalizador GAPDH.

\section{Histología}

Se tomó una biopsia de $5 \times 5 \mathrm{~mm}$ en el interior de la zona de la herida al inicio del tratamiento y otra al séptimo día, ambas fijadas en formol al $10 \%$ en PBS y luego incluidas en parafina, y cortadas en espesores de $5 \mu \mathrm{m}$. Los cortes se montaron en laminillas de vidrio y después se desparafinaron, hidrataron, tiñeron con hematoxilina de Harris y contrastaron con eosina. Por último, las preparaciones se aclararon y montaron con resina de metacrilato/acrilato (Entellan Merck, Alemania).

\section{Respuesta de la lesión}

La recuperación de la lesión se determinó al plantear el índice úlcera, que se interpretó por el porcentaje del área de la herida abierta remanente con respecto al área de la herida al inicio del tratamiento. También se expresó la tasa de recuperación $\left(T_{r}\right)$ de la herida como el cociente $T_{r}=A_{r} / t_{r}$, en el cual $A_{r}$ es el valor del área recuperada $\left(\mathrm{cm}^{2}\right)$ durante el tratamiento, $\mathrm{y}_{\mathrm{r}}$ los días que duró la recuperación del área. Las extensiones de las áreas ulceradas y recuperadas se evaluaron mediante el análisis de imágenes con el programa ImageJ.

\section{Condición inflamatoria}

Para determinar la condición inflamatoria de la paciente, se evaluó el fenotipo TH1/TH2 en muestras de sangre periférica antes y después del tratamiento, a través del kit II Th1/Th2 (CBA, EE.UU.), que identifica citocinas mediadoras de la inflamación: interferón gamma (IFN- $\gamma$ ), interleucina 2 (IL-2), factor de necrosis tumoral alfa (TNF- $\alpha$ ), interleucina 4 (IL-4), interleucina 6 (IL-6) e interleucina 10 (IL-10). La evaluación de las células se realizó en un citómetro de flujo FACS Di$v^{T M}$ III (BD Immunocytometry Systems, EE.UU.) y para el análisis de los datos se utilizó el software $B D^{T M}$ Cytometric Bead Array Software, versión 1.4.

\section{Resultados}

\section{Células madre mesenquimales}

Las células de la gelatina de Wharton se expandieron, seleccionaron y aislaron (Fig. 1). El cultivo presentó morfología característica de CMM con semejanza a fibroblastos (células pequeñas, largas y estrechas con un núcleo redondo, grande y céntrico) (Fig. 1A). Por otra parte, en presencia del medio osteogénico las células respondieron con un proceso de diferenciación celular al formar depósitos de calcio extracelulares teñibles con rojo de alizarina (Fig. 1B). Asimismo, en presencia de un medio adipogénico, las células se diferenciaron y formaron vesículas intracelulares positivas a la tinción con rojo oleoso (Fig. 1C). La PCR en tiempo real mostró que las CMM manifestaron expresión génica de los marcadores de superficie CD73, CD90 y CD105 y no de los marcadores hematopoyéticos CD34, CD45 y HLA-DR (Fig. 1D), lo cual concuerda con las características señaladas por la ISCT ${ }^{26}$.

\section{Respuesta de la lesión al tratamiento}

La aplicación cutánea de las CMM sobre la lesión recuperó tejido al inducir desbridamiento y cicatrización (Fig. 2). Al séptimo día de tratamiento, la lesión mostró reducción de su extensión, profundidad y grosor al presentar bordes mejor definidos, con una mayor extensión de piel en los márgenes y una capa celular superficial, típica señal de cicatrización (Figs. 2A y B); además, los sitios de toma de biopsia se rellenaron rápidamente con tejido. Por otra parte, el área abierta se redujo en $70 \%$ (Fig. 2C) y con una tasa de cierre más rápida (Fig. 2D) en los primeros siete días del tratamiento, mientras que en la segunda aplicación el índice de úlcera se redujo hasta un $80 \%$ (Fig. 2C).

\section{Histopatología}

En los cortes histológicos de la herida sin tratar se mostró un estado leve de inflamación (Fig. 3), pero sin presentar signos de infección. Se identificó un notorio infiltrado leucocitario perivascular y edema en la evaluación inicial; sin embargo, después de la primera aplicación y ya en la segunda dichos infiltrados decrecieron y pudo identificarse la formación de nuevos vasos sanguíneos y fibras de colágena revelados por la tinción de hematoxilina (Figs. 3A y B). La cuenta de leucocitos en el tejido mostró una disminución significativa mayor de $20 \%$ después del tratamiento con CMM (Fig. 3C).

\section{Atenuación del estado proinflamatorio con células madre mesenquimales}

En muestras sanguíneas se analizó la presencia de citocinas proinflamatorias y antiinflamatorias antes y 


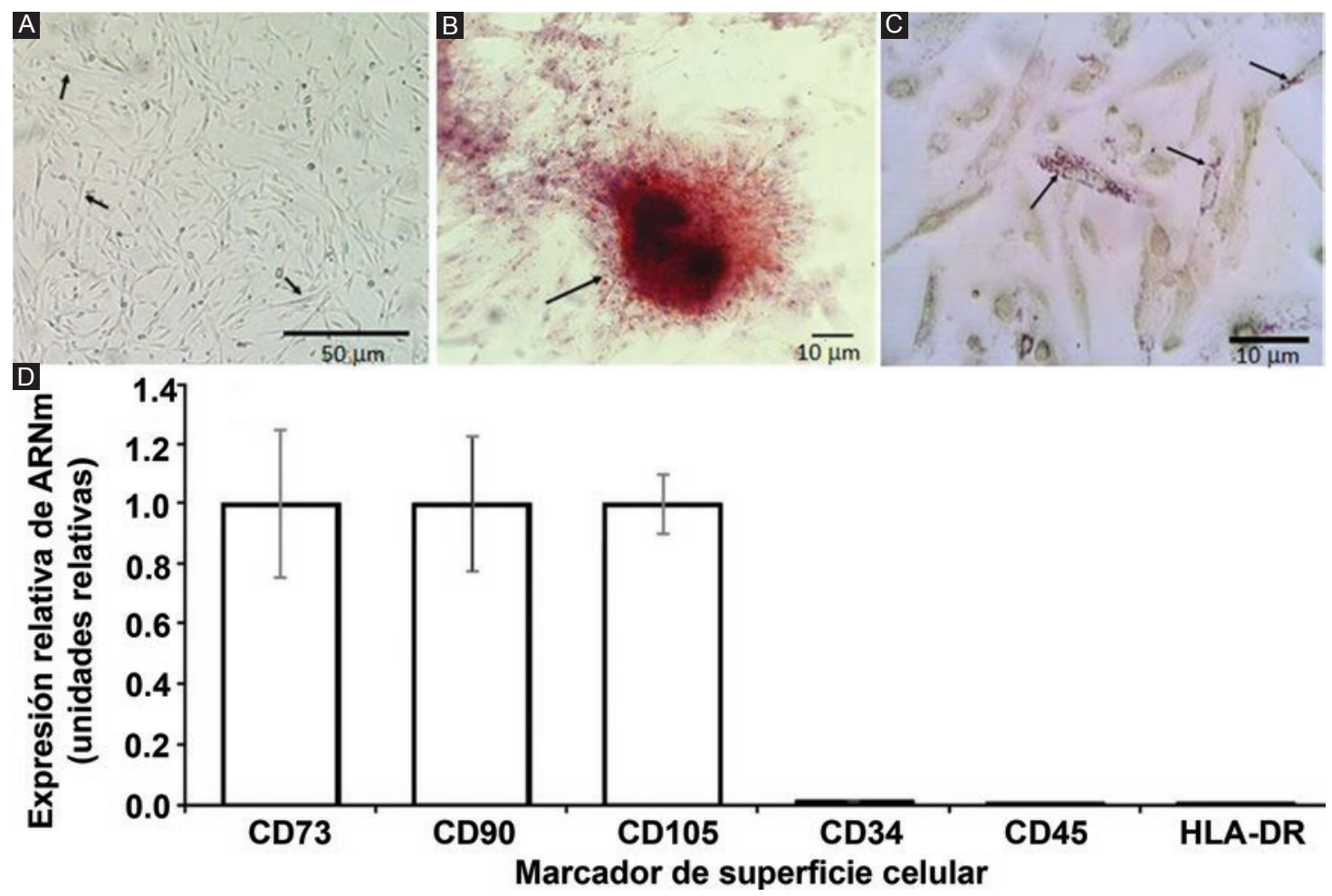

Figura 1. Obtención y caracterización de las CMM de gelatina de Wharton. A: Cultivos de CMM de gelatina de Wharton en el sexto pase; B: Células en medio de diferenciación osteogénica, $\uparrow$, acúmulos de $\mathrm{Ca}^{++}$y fosfato extracelular teñidos con rojo de alizarina; C: Células en medio de diferenciación adipogénico, $\uparrow$, con vesículas lipídicas intracelulares teñidas con rojo oleoso; D: Análisis de la expresión génica relativa. Microscopio de campo claro invertido Oxion (PCE Instruments, Chile).

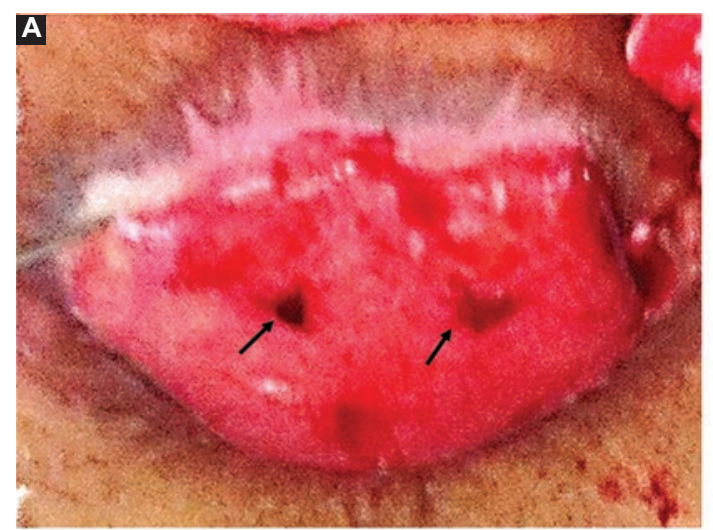

C

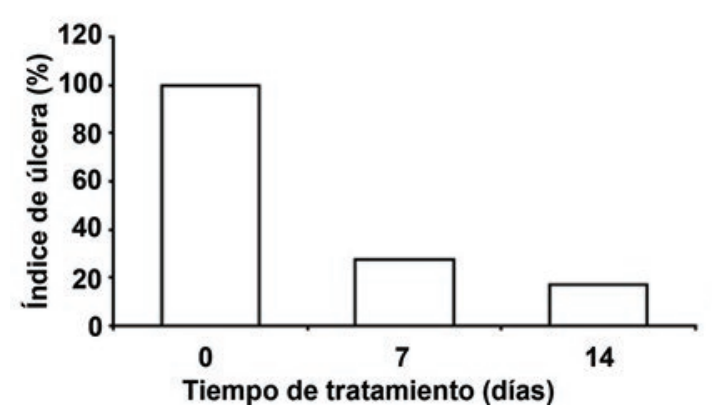

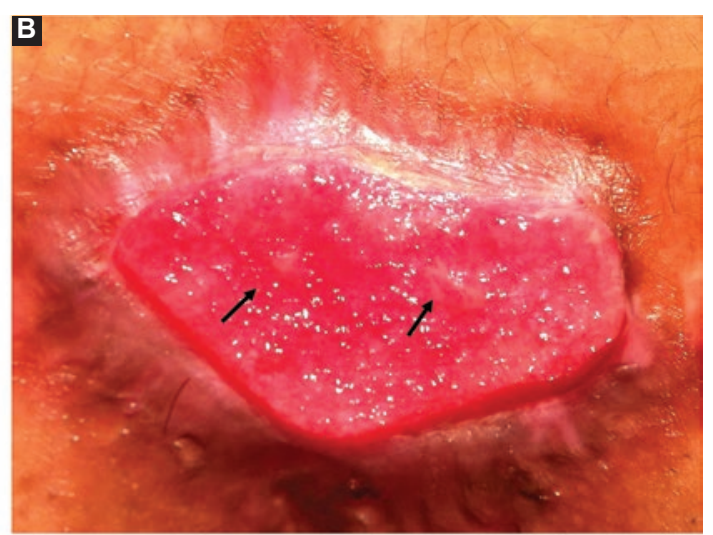

D

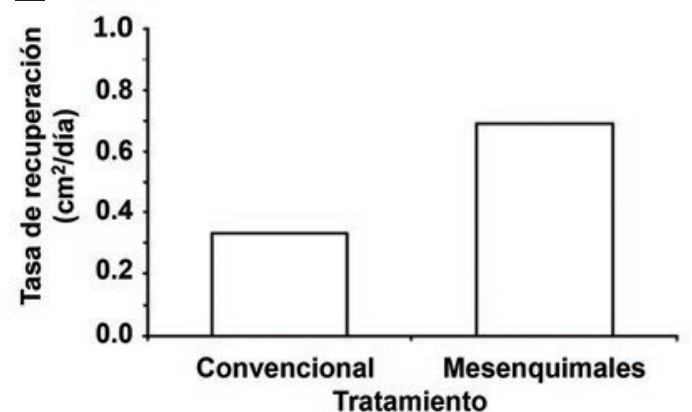

Figura 2. Respuesta de la lesión. A: Aspecto de la herida antes del tratamiento; B: Aspecto de la herida siete días después del primer tratamiento, $\uparrow:$ sitios de biopsias; C: Índice de úlcera con respecto al tiempo del tratamiento; D: Tasa recuperación. Microscopio de campo claro invertido Oxion (PCE Instruments, Chile). 

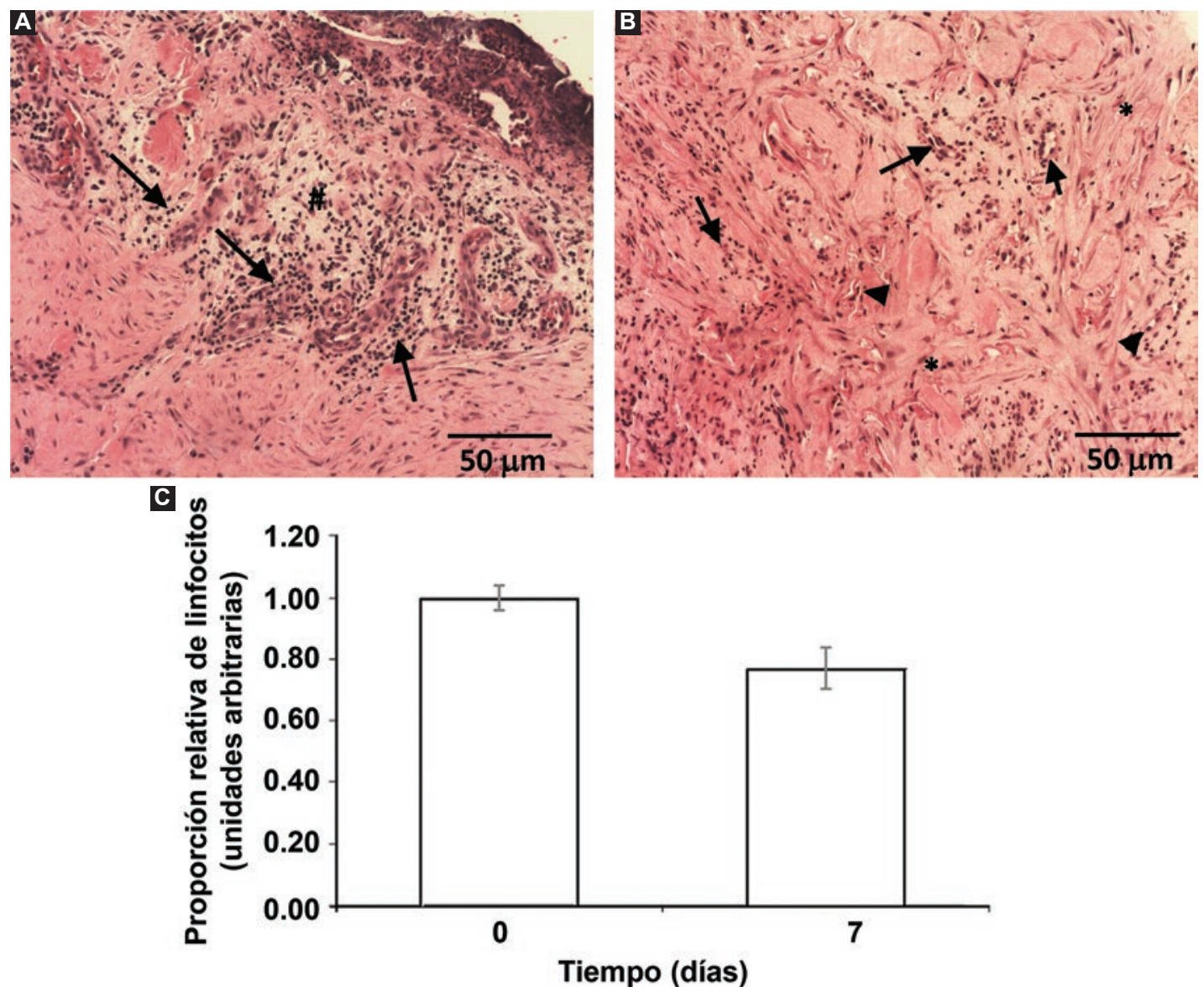

Figura 3. Recuperación de la lesión. A: Cortes histológicos antes del tratamiento; B: despúes de siete días del primer tratamiento, $\uparrow:$ infiltrado linfocitario perivascular, \#: edema, $\mathbf{\Delta}$ : formación de vasos, *: fibras de colágena; C: Conteo leucocitario en tejido prueba $t$ de Student para muestras relacionadas, $p<0.05$. Microscopio de campo claro invertido Oxion (PCE Instruments, Chile).

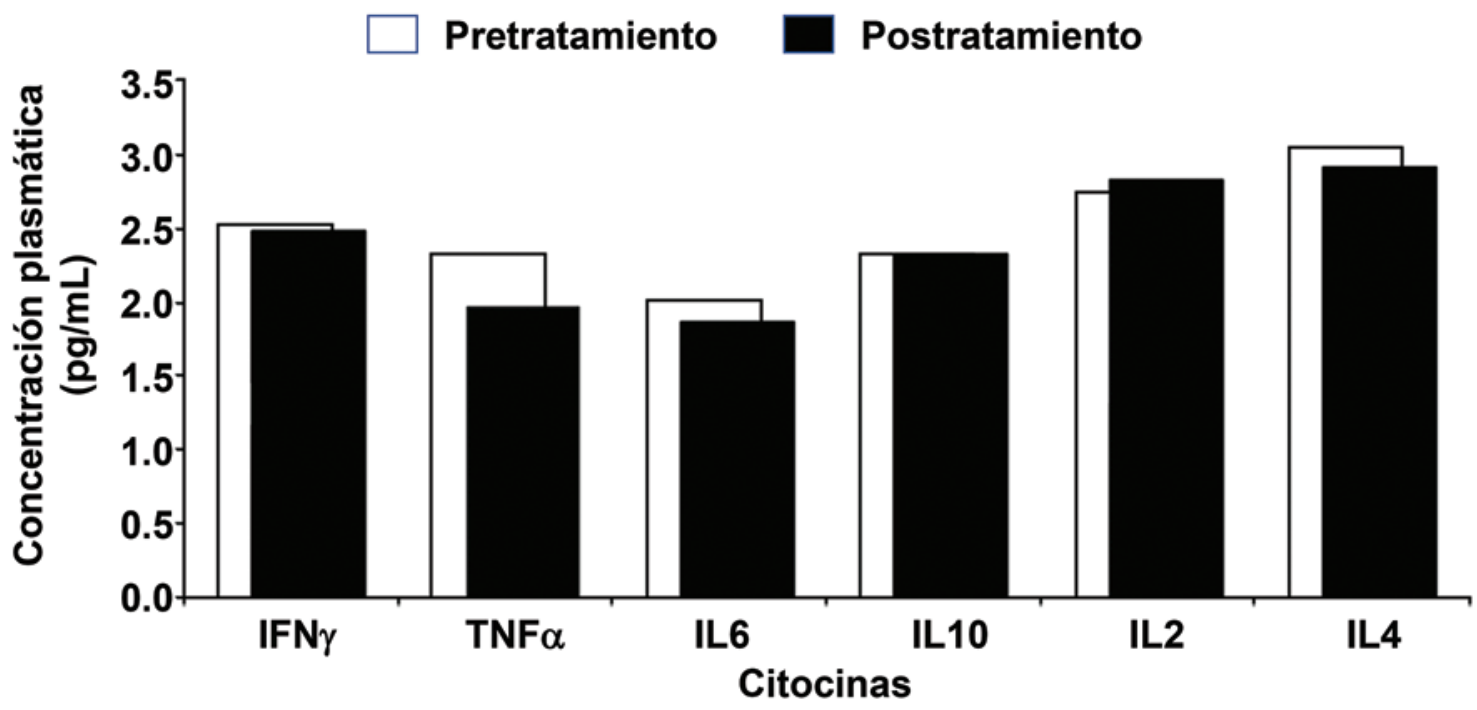

Figura 4. Cuantificación de citocinas en plasma de sangre periférica antes y después de siete días del primer tratamiento celular.

después del tratamiento celular (Fig. 4) y se observó que el tratamiento con CMM propició una disminución de las citocinas proinflamatorias TNF $\alpha$, IL-6, IFN $\gamma$ y la citocina antiinflamatoria IL-4. Pese a ello, el tratamiento incrementó el número de las citocinas antiinflamatorias IL-2 e IL-10. 


\section{Discusión}

La herida con tratamiento convencional de tres meses no sanó, ya que se encontraba en condiciones no aptas para repararse debido a un proceso de cicatrización deficiente. Esto pudo deberse a un estado general desfavorable (estrés, hábitos de vida, entre otros $)^{28}$ en relación con su inmunología, metabolismo, estado nutricional y emocional ocasionado por el sobrepeso que lleva a los pacientes a someterse a una cirugía de dermolipectomía ${ }^{29}$. Debido a la persistencia de la abertura de la lesión, la paciente fue elegible para recibir tratamiento con CMMGW de cordón umbilical, que se ha señalado como una opción terapéutica ${ }^{27}$.

Las células que recibió la paciente fueron CMM y cumplieron con los criterios mínimos establecidos por la ISCT para el tratamiento celular, como la adherencia y forma del fibroblasto, lo que hizo posible su selección en cultivo como se indica para las $\mathrm{CMM}^{26}$. En cuanto a las proteínas marcadoras de superficie CD73, CD90 y CD105, su expresión se reveló por la presencia del RNAm correspondiente, mientras que la expresión de CD34, CD45 y HLA-DR fue reducida o nula (como se esperaba), por lo que se satisficieron todas las características de CMM propuestas por la $\mathrm{ISCT}^{26}$.

En otro aspecto, las células mostraron capacidad de diferenciación osteogénica y produjeron acúmulos que se tiñeron con rojo de alizarina, que es afín a los depósitos de calcio y fosfato, lo que indicó que las células se diferenciaron en osteoblastos. Asimismo, las células en medio adipogénico se diferenciaron en células que mostraron acumulación de vesículas citoplásmicas positivas a la tinción con rojo oleoso, que reveló los lípidos característicos de adipocitos. Ambas pruebas confirmaron la plasticidad de las CMM como el tercer criterio de la ISCT $20,26,30$.

Los resultados mostraron que la administración de CMM mejoró la recuperación de la úlcera en un tiempo menor al que toma el tratamiento convencional, probablemente porque se favoreció la cicatrización, que se relaciona con las fases bien coordinadas entre la inflamación, la proliferación celular y la remodelación de la lesión ${ }^{31,32}$.

En cuanto a la evaluación médica de la lesión, se pudo observar que el tratamiento celular indujo un proceso de desbridamiento natural que facilita la fase proliferativa del proceso de cicatrización debido a la liberación in situ de factores como TNF $\alpha$, IFN $\gamma$ y IL-6 ${ }^{33}$, lo cual favorece el desplazamiento, atracción e infiltración de plaquetas y queratinocitos nativos que remodelan la zona de la lesión. Los macrófagos también tienen una función muy importante, dado que éstos desbridan y remodelan la zona afectada ${ }^{28,32-34}$ en conjunto con otros leucocitos. En la fase proliferativa también se desarrollan fenómenos de angiogénesis, los cuales fueron evidentes en los cortes histológicos. La vascularización favorecida en la lesión pudo promover la irrigación sanguínea y el paso de oxígeno y nutrientes, lo que contribuye a la formación de fibras de colágena en la disposición adecuada para formar una matriz extracelular de soporte que llevó a la fase de maduración y remodelación del tejido ${ }^{28,34}$.

En los cortes histológicos se reconoció la presencia de fibras de colágena formadas de novo por el tratamiento (característico de la fase proliferativa de la cicatrización), lo que precede a la fibroplastia y formación de tejido granular; esta nueva matriz extracelular proporciona el soporte celular para estimular la epitelización y contracción de la herida ${ }^{31,32}$. Lo anterior llevó a la reducción tanto del área como de la profundidad de la herida, en especial de los sitios donde se tomó la biopsia, lo que indicó regeneración ${ }^{28,34}$.

Tal y como se ha descrito en muchos tratamientos en los que se administran células mesenquimales, no se produjo rechazo inmunológico debido a que las CMM son de baja inmunogenicidad, y con propiedades inmunomoduladoras que no inducen la expresión del MHC clase $\|^{19,31}$. Dichas propiedades se vieron reflejadas al detectar una disminución de las citocinas proinflamatorias TNF $\alpha$, IFN $\gamma$ e IL- 6 en sangre, mientras que las cantidades de las moléculas antiinflamatorias IL-2 e IL-10 se mantuvieron; no obstante, se detectaron valores bajos de IL-4. Esto coincide con lo observado en la inhibición de la proliferación de esplenocitos de ratón y linfocitos humanos, en la cual se suprime además la secreción de otras citocinas inflamatorias como el IFN- $\gamma^{16,20,22-25}$.

Los autores proponen que el cambio en el estado de inflamación es efecto del tratamiento con CMMGW, como se ha demostrado en otras lesiones crónicas $^{34}$; no obstante, con estos resultados sería importante realizar un análisis estadístico para asegurar una relación causa-efecto.

El nuevo equilibrio del estado de inflamación creado por el tratamiento celular, como se ha documentado en otros trabajos, posibilitó la proliferación celular, la angiogénesis y el desarrollo de una matriz extracelular de soporte y diferenciación para las mismas CMM, así como para otras células. Del mismo modo, favoreció el ingreso de elementos y factores procicatrizantes y 
activó la nutrición, oxigenación y eliminación de desechos que promovieron la reparación del tejido, de tal modo que mejoró la apariencia de la herida al igual que las condiciones que mantenían el estado persistente de la úlcera ${ }^{16,28,31-35}$.

\section{Conclusiones}

El tratamiento con células madre mesenquimales de gelatina de Wharton es factible para tratar una úlcera crónica persistente derivada de una dermolipectomía de mal pronóstico y difícil manejo, lo que representa una interesante alternativa médica con potencial para muchos otros tipos de lesiones, en los cuales se permita la regulación de los procesos inmunológicos, quimiotácticos y modeladores del tejido y una baja inmunogenicidad. Esta modalidad permite que el paciente controle sus procesos de cicatrización con menos asistencia a consultas, lo que implica en términos de costo y beneficio un menor gasto en servicios de salud a largo plazo y una reducción del tiempo de convalecencia.

\section{Agradecimientos}

Los autores agradecen a la Escuela Superior de Medicina del Instituto Politécnico Nacional, por el apoyo económico, equipos y espacios. Al Instituto de Seguridad Social del Estado de México y Municipios (ISSEMYM) la canalización, evaluación y seguimiento de la paciente. $Y$ al Banco Central de Sangre Centro Médico Nacional La Raza, IMSS, por proveer el material biológico.

\section{Financiamiento}

Los autores declaran que el presente trabajo no contó con financiamiento externo. Los recursos empleados para su desarrollo dependieron del proyecto individual SAPPI 20171880 del Instituto Politécnico Nacional.

\section{Conflicto de intereses}

Los autores declaran no tener ningún conflicto de intereses.

\section{Responsabilidades éticas}

Protección de personas y animales. Los autores declaran que los procedimientos seguidos están apegados a las normas éticas del comité de experimentación humana responsable y de acuerdo con la Asociación Médica Mundial y la Declaración de Helsinki.

Confidencialidad de los datos Los autores declaran que han seguido los protocolos de su centro de trabajo sobre la publicación de datos de pacientes.

Derecho a la privacidad y consentimiento informado Los autores han obtenido el consentimiento informado de los pacientes o sujetos referidos en el artículo. Este documento se halla en poder del autor de correspondencia.

\section{Bibliografía}

1. Gentileschi S, Servillo M, D'Ettorre M, Salgarello M. Abdominal subcutaneous mass after laser-assisted lipolysis and immediate multiple treatments with a dual-wavelength laser, vacuum and massage device. Aesthet Surg J. 2016;36:NP144-149.

2. Martínez-Teixido L, Serra-Mestre JM, Serra-Renom JM. A new technique for creating a neo-umbilicus in abdominoplasty. J Plast Reconstr Aesthet Surg. 2017;70:1760-1767.

3. García-García ML, Martín-Lorenzo JG, Campillo-Soto A, Torralba-Martínez JA, Liron-Ruiz R, Miguel-Perello J, et al. Complications and level of satisfaction after dermolipectomy and abdominoplasty post-bariatric surgery. Cir Esp. 2014;92:254-260.

4. Merle R, Serror K, Marco O, Chaouat M, Teissier S, Mimoun M, et al. Study of satisfaction concerning the navel after abdominal dermolipectomy with transposition: a report of 96 cases. Ann Chir Plast Esthet. 2017.

5. Davis FM, Kimball A, Boniakowski A, Gallagher K. Dysfunctional wound healing in diabetic foot ulcers: new crossroads. Curr Diab Rep. 2018;18:2.

6. Lantis JC, $2^{\text {nd }}$, Marston WA, Farber A, Kirsner RS, Zhang Y, Lee TD, et al. The influence of patient and wound variables on healing of venous leg ulcers in a randomized controlled trial of growth-arrested allogeneic keratinocytes and fibroblasts. J Vasc Surg. 2013;58:433-439.

7. Greer N, Foman N, Dorrian J, Fitzgerald P, MacDonald R, Rutks I, et al. Advanced wound care therapies for non-healing diabetic, venous, and arterisl ulcers: a systematic review. Washington DC: Departments of Veterans Affairs, 2012.

8. Giuggioli D, Manfredi A, Lumetti F, Sebastiani M, Ferri C. Cryoglobulinemic vasculitis and skin ulcers. Our therapeutic strategy and review of the literature. Semin Arthritis Rheum. 2015;44:518-526.

9. Sen CK, Gordillo GM, Roy S, Kirsner R, Lambert L, Hunt TK, et al. Human skin wounds: a major and snowballing threat to public health and the economy. Wound Repair Regen. 2009;17:763-771.

10. Hamish M, Gohel MS, Shepherd A, Howes NJ, Davies AH. Variations in the pharmacological management of patients treated with carotid endarterectomy: a survey of European vascular surgeons. Eur J Vasc Endovasc Surg. 2009;38:402-407.

11. Gohel MS, Davies AH. Pharmacological agents in the treatment of venous disease: an update of the available evidence. Curr Vasc Pharmacol. 2009;7:303-308

12. Tenci M, Rossi S, Bonferoni MC, Sandri G, Boselli C, Di Lorenzo A, et al. Particulate systems based on pectin/chitosan association for the delivery of manuka honey components and platelet lysate in chronic skin ulcers. Int J Pharm. 2016;509:59-70.

13. Pop MA, Almquist BD. Biomaterials: a potential pathway to healing chronic wounds? Exp Dermatol. 2017;26:760-763.

14. Badiavas EV, Falanga V. Treatment of chronic wounds with bone marrow-derived cells. Arch Dermatol. 2003;139:510-516.

15. Chen MY, Lie PC, Li ZL, Wei X. Endothelial differentiation of Wharton's jelly-derived mesenchymal stem cells in comparison with bone marrow-derived mesenchymal stem cells. Exp Hematol. 2009;37:629-640.

16. Zhou C, Yang B, Tian Y, Jiao H, Zheng W, Wang J, et al. Immunomodulatory effect of human umbilical cord Wharton's jelly-derived mesenchymal stem cells on lymphocytes. Cell Immunol. 2011;272:33-38.

17. Bongso A, Fong CY, Gauthaman K. Taking stem cells to the clinic: major challenges. J Cell Biochem. 2008;105:1352-1360.

18. Gao LR, Zhang NK, Ding QA, Chen HY, Hu X, Jiang S, et al. Common expression of stemness molecular markers and early cardiac transcription factors in human Wharton's jelly-derived mesenchymal stem cells and embryonic stem cells. Cell Transplant. 2013;22:1883-1900.

19. Weiss ML, Anderson C, Medicetty S, Seshareddy KB, Weiss RJ VanderWerff I, et al. Immune properties of human umbilical cord Wharton's jelly-derived cells. Stem Cells. 2008;26:2865-2874. 
20. Li X, Bai J, Ji X, Li R, Xuan Y, Wang Y. Comprehensive characterization of four different populations of human mesenchymal stem cells as regards their immune properties, proliferation and differentiation. Int J Mol Med. 2014;34:695-704.

21. Le Blanc K, Tammik L, Sundberg B, Haynesworth SE, Ringden O. Mesenchymal stem cells inhibit and stimulate mixed lymphocyte cultures and mitogenic responses independently of the major histocompatibility complex. Scand J Immunol. 2003:57:11-20.

22. Magatti M, De Munari S, Vertua E, Gibelli L, Wengler GS, Parolini O. Human amnion mesenchyme harbors cells with allogeneic T-cell suppression and stimulation capabilities. Stem Cells. 2008;26:182-192.

23. Asari S, Itakura S, Ferreri K, Liu CP, Kuroda Y, Kandeel F, et al. Mesenchymal stem cells suppress B-cell terminal differentiation. Exp Hematol. 2009;37:604-615.

24. Spaggiari GM, Capobianco A, Abdelrazik H, Becchetti F, Mingari MC, Moretta L. Mesenchymal stem cells inhibit natural killer-cell proliferation, cytotoxicity, and cytokine production: role of indoleamine 2,3-dioxygenase and prostaglandin E2. Blood. 2008:111:1327-1333.

25. English K, Barry FP, Mahon BP. Murine mesenchymal stem cells suppress dendritic cell migration, maturation and antigen presentation. Immunol Lett. 2008;115:50-58.

26. Dominici M, Le Blanc K, Mueller I, Slaper-Cortenbach I, Marini F, Krause D, et al. Minimal criteria for defining multipotent mesenchymal stromal cells. The International Society for Cellular Therapy position statement. Cytotherapy. 2006;8:315-317.

27. Benítez-Arvizu G, Palma-Laral,Vázquez-Campos R, Sesma-Villalpando RA, Parra-Barrera A, Gutiérrez-Iglesias G. Autologous mesenchymal stem cells and cutaneus autograft as a treatment for chronic ulcer secondary to diabetes mellitus 2. Cir Cir. 2015;83:532-536

28. Midwood KS, Williams LV, Schwarzbauer JE. Tissue repair and the dynamics of the extracellular matrix. Int J Biochem Cell Biol. 2004; 36:1031-1037.

29. Sam S, Mazzone T. Adipose tissue changes in obesity and the impact on metabolic function. Transl Res. 2014;164:284-292.

30. Pittenger MF, Mackay AM, Beck SC, Jaiswal RK, Douglas R, Mosca JD, et al. Multilineage potential of adult human mesenchymal stem cells. Science. 1999;284:143-147.

31. Stadelmann WK, Digenis AG, Tobin GR. Physiology and healing dynamics of chronic cutaneous wounds. Am J Surg. 1998;176:26S-38S.

32. Iba $Y$, Shibata A, Kato M, Masukawa T. Possible involvement of mast cells in collagen remodeling in the late phase of cutaneous wound healing in mice. Int Immunopharmacol. 2004;4:1873-1880.

33. Chapel A, Bertho JM, Bensidhoum M, Fouillard L, Young RG, Frick J, et al. Mesenchymal stem cells home to injured tissues when co-infused with hematopoietic cells to treat a radiation-induced multi-organ failure syndrome. J Gene Med. 2003:5:1028-1038.

34. Maxson S, López EA, Yoo D, Danilkovitch-Miagkova A, Leroux MA. Concise review: role of mesenchymal stem cells in wound repair. Stem Cells Transl Med. 2012;1:142-149.

35. Chang HY, Sneddon JB, Alizadeh AA, Sood R, West RB, Montgomery K, et al. Gene expression signature of fibroblast serum response predicts human cancer progression: similarities between tumors and wounds. PLoS Biol. 2004;2:E7. 